\title{
Diagnosis of LIB Degradation using Estimating Cell Resistance for Hybrid Electric Vehicles
}

\author{
Jin-Ho Park*, Woo-Suk Sung** and Byoung Kuk Lee ${ }^{\dagger}$
}

\begin{abstract}
To get computationally efficient algorithm which can diagnose the cell degradation by estimating the cell resistance in a battery management system (BMS), the ordinary least squares estimator (LMS filter) is employed to identify the parameter in the simple resistance model in the form of a first-order polynomial. As a result, the determined parameter estimate, the slope in the model, can be adapted to changes in the battery resistance. The devised algorithm is validated by using the cells with different state-of-health. These cells are aged at the elevated temperature over 24 weeks and tested at every four weeks to monitor changes in the cell resistance. Consequently, the devised algorithm can distinguish between the cell resistances which increase by 2 to $4 \%$ at every month.
\end{abstract}

Keywords: Lithium-ion batteries, Battery management systems, Least squares estimator, Internal resistance, Degradation

\section{Introduction}

Recently, the electrified powertrain holds immense potential for increasing fuel economy while reducing or even eliminating emissions. Among several batteries, lithium-ion batteries (LIBs) are mainly applicable for the electrified powertrain as energy storage solution. In order to manage a multiple of LIB cells simultaneously, a LIB pack should be controlled by a battery management system (BMS). A BMS consists of hardware and software for battery management, primarily including algorithms for identifying battery states, such as state-of-charge (SOC), state-of-health ( $\mathrm{SOH})$ and resistance. By using a BMS, a LIB pack can be run within its safe operating area which is typically restricted by the applied current, SOC and temperature.

In case of the battery for hybrid electric vehicles (HEVs), the power is more critical because that the extractable power from the battery will determine the vehicle's fuel economy as well as acceleration and the available power to and from the battery by charging and discharging, respectively, is primarily limited by the internal resistance of a battery.

For the purpose of estimating the battery resistance, a battery model and its state and/or parameter estimator are mainly used. As for battery models, (semi-)empirical models based on equivalent circuit [1-7] and electrochemical models $[8,9]$ are widely applied. Despite potential benefits from informative states and/or parameters of the

$\dagger$ Corresponding Author: Department of Electrical and Computer Engineering, Sungkyunkwan University, Korea. (bkleeskku@skku.edu)

* Department of Electrical and Computer Engineering, Sungkyunkwan University, Korea. (pjh001@gmail.com)

** Hyundai Motor Company in R \& D Division, Korea. (wsung1@gmail.com)

Received: December 24, 2015; Accepted: March 18, 2016 electrochemical models, its implementation into the computationally light BMS is difficult. As for a state and/or parameter estimator, two different estimators are mainly used, such as the Kalman filter $[1,2]$ and the least mean squares (LMS) filter [3-7]. With the respect of computational efficiency in BMS, the LMS filter is significantly effective to the Kalman filter due to the absence of complex matrix calculations such as inversions. Furthermore, the recursive LMS filters do not require large memory footprint.

Considering the above-mentioned problems, the computationally efficient algorithm is devised for estimating the battery resistance by only using the limited computational resources of the BMS. Toward this end, the devised algorithm employs a simple resistance model in the form of a linear equation, instead of the non-linear ECMs. The devised algorithm also exploits the ordinary least squares estimator in order to identify a model parameter that represents the battery resistance varying with the SOC, temperature and aging time. For reliable estimation of the battery resistance, the available ranges of the SOC and temperature are specified. Furthermore, a preferred limit of the correlation coefficient is set such that the battery resistance can be estimated only if a strong correlation between the voltage and the current is found. By doing this, the determined parameter estimate, that is, the slope in the model, can be adapted to changes in the battery resistance.

In consequence, the estimated resistance can act as a battery degradation index. Without the degradation index, to use the battery for HEVs as an example, the required power at end-of-life (EOL) is normally set to be about $80 \%$ of the required power at the beginning-of-life (BOL). This implies that even brand-new batteries are actually in service only with about $80 \%$ of the maximum power. 
This underutilization should cause the battery to be oversized and expensive. By means of the degradation index represented by the estimated resistance, the available power can vary according to battery degradation such that the battery is no longer conservatively operated.

\section{Design of Proposed Algorithm}

This section describes the design of the proposed algorithm for the estimation of the battery resistance in more detail. Linear regression is primarily used for estimating the battery resistance. This is realized by means of the ordinary least squares estimator, in other words, the LMS filter. Basically, regression fits a model to data. As shown in Fig. 1, the resistance model here is in the form of a first-order polynomial so that it can be assumed to be a straight line:

$$
y=\alpha+\beta x+\varepsilon
$$

which gives a best fit for data. This means that the LMS filter finds the parameter values that minimize the objective function Q. Thus, the sum-of-squared (SS) error $\varepsilon$ between the model and data is expressed as:

$$
\min _{\alpha, \beta} Q=\sum_{i=1}^{n} \varepsilon^{2}=\sum_{i=1}^{n}\left(y_{i}-\alpha-\beta x_{i}\right)^{2}
$$

The model involves the y-intercept $\alpha$ and the slope $\beta$, as shown in Eq. (1). As also shown in Fig. 1, the data include the cell voltage $\mathrm{V}$ along with y coupled with the current $\mathrm{I}$ along with $\mathrm{x}$. Basically, the cell voltage varies in response to the current such that the current is an independent variable while the voltage is a dependent variable. The internal resistance $\mathrm{R}$ can then be estimated by dividing the voltage with the current, which is called Ohm's law. Therefore, the parameter in the model, the slope, can reflect the cell resistance. The model can be determined by taking the partial derivative with respect to $\alpha$ and $\beta$, as follows. In the derivation, Eq. (2) is denoted as SS. First, solving for $\alpha$,

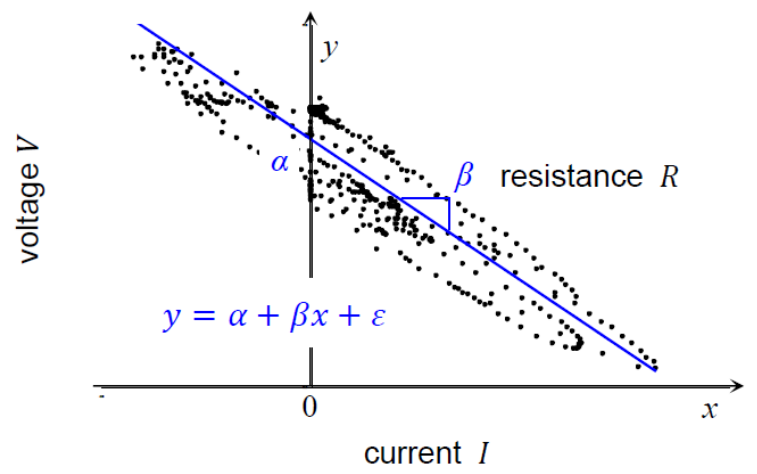

Fig. 1. Simple resistance model in the form of a linear equation

$$
\begin{gathered}
\frac{\partial S S}{\partial \alpha}=\sum_{i=1}^{n}\left(-2 y_{i}+2 \beta x_{i}+2 \alpha\right)=0 \\
\sum_{i=1}^{n} y_{i}-\sum_{i=1}^{n} \beta x_{i}-n \alpha=0
\end{gathered}
$$

which yields

$$
\alpha=\frac{1}{n}\left(\sum_{i=1}^{n} y_{i}-\beta \sum_{i=1}^{n} x_{i}\right)
$$

Eq. (5) can be rewritten, more succinctly, as:

$$
\alpha=\overline{y_{l}}-\beta \overline{x_{l}}
$$

where

$$
\overline{y_{l}}=\frac{1}{n} \sum_{i=1}^{n} y_{i}
$$

Then, solving for $\beta$,

$$
\begin{aligned}
& \frac{\partial S S}{\partial \beta}=\sum_{i=1}^{n}\left(-2 y_{i} x_{i}+2 \alpha x_{i}+2 \beta x_{i}^{2}\right)=0 \\
& \sum_{i=1}^{n} y_{i} x_{i}-\sum_{i=1}^{n} \alpha x_{i}-\sum_{i=1}^{n} \beta x_{i}^{2}=0
\end{aligned}
$$

By substituting Eq. (6), Eq. (9) becomes:

$$
\begin{aligned}
& \sum_{i=1}^{n} y_{i} x_{i}-\left(\overline{y_{l}}-\beta \overline{x_{l}}\right) \sum_{i=1}^{n} x_{i}-\sum_{i=1}^{n} \beta x_{i}^{2}=0 \\
& \beta\left(\overline{x_{l}} \sum_{i=1}^{n} x_{i}-\sum_{i=1}^{n} x_{i}^{2}\right)=-\sum_{i=1}^{n} y_{i} x_{i}+\bar{y}_{l} \sum_{i=1}^{n} x_{i}
\end{aligned}
$$

which finally yields

$$
\beta=\frac{n \sum_{i=1}^{n} x_{i} y_{i}-\sum_{i=1}^{n} x_{i} \sum_{i=1}^{n} y_{i}}{n \sum_{i=1}^{n} x_{i}^{2}-\left(\sum_{i=1}^{n} x_{i}\right)^{2}}
$$

In essence, the estimation of the battery resistance is carried out as follows. First, the cell voltage and current data is gathered. The cell voltage is measured with the current at every 100 milliseconds. This continues for one minute, resulting in 600 pairs of voltage and current measurements accumulated. It is noted that all of the measurements need not be stored simultaneously. Rather, only the new measurement is stored and then added to the cumulative sum of the last measurements. In this work, the number of the measurements is set to be 600 ; yet, it is not necessarily. Too small numbers could lead to unreliable regression, if they capture transient battery behaviors. Also, too small numbers are inappropriate in that changes in the battery resistance are inherently tardy. Too large numbers will cause trouble in the other way. Too large numbers should increase the cumulative sum of the measurements as Eqs. (4), (11), which is computationally costly particularly considering the limited computational resources of the BMS. Next, model parameters are estimated from the data. This follows the predetermined procedure, as described in Eqs. (3) - (12). For instance, throughout the driving cycle for the duration of 2,140 seconds, 35 data sets and thus 35 estimates are produced in series. That is, a new estimate is 

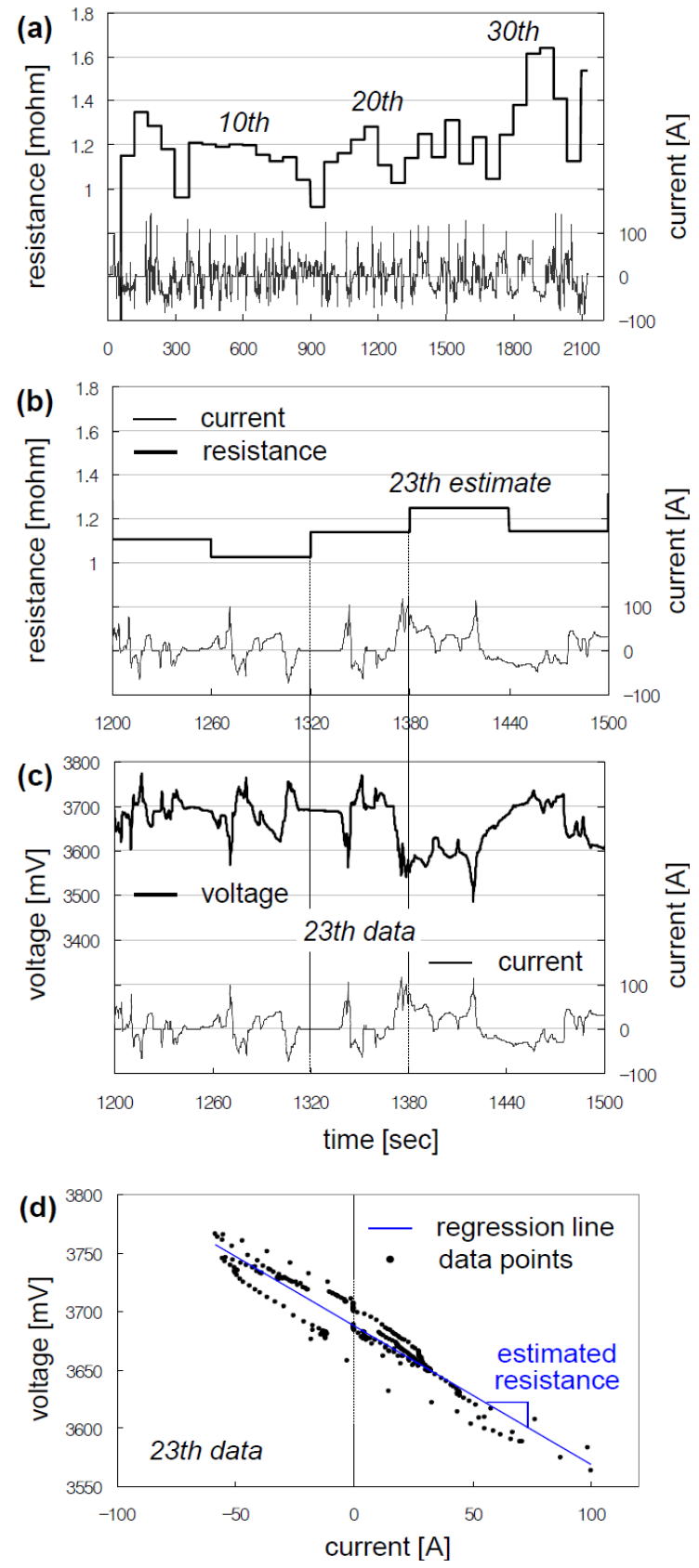

Fig. 2. Estimation of the battery resistance by using a HEV driving cycle

created at 60-second intervals as shown in Fig. 2(a). The insets are useful to take a closer look at the timeslot inbetween 1,320 to 1,380 seconds, which is the 23th data set creating the 23th estimate as shown in Figs. 2(b) and (c). Within the 23th data set, the resistance model is fitted, resulting in the least squares regression line whose slope is indicative of the resistance estimate as shown in Fig. 2(d). However, not all of them can be qualified as a valid parameter value, that is to say, the effective battery resistance. Therefore, the parameter estimates are then selected. For this, in addition to the slope, the correlation coefficient $r$ is used as another parameter. The correlation coefficient is a measure of the strength and the direction of a linear relationship between two variables; here, an independent variable is the current whereas a dependent variable is the voltage. The correlation coefficient is the covariance of two variables divided by the product of their individual standard deviations. This can be described as:

$$
r=\frac{\sum_{i=1}^{n}\left(x_{i}-\bar{x}\right)\left(y_{i}-\bar{y}\right)}{\sqrt{\sum_{i=1}^{n}\left(x_{i}-\bar{x}\right)^{2} \sum_{i=1}^{n}\left(y_{i}-\bar{y}\right)^{2}}}
$$

Generally, the correlation coefficient ranges from -1 to 1 . If two variables have a positive correlation then it spans from 0 to 1 . If two variables have a negative correlation then it spans from -1 to 0 . The positive correlation means if an independent variable increase then a dependent variable also increases. The negative correlation goes the opposite way around. These signs are related to the direction of a linear relationship; however, it is not important in this work because the direction can vary according to the sign convention. Here, unless otherwise noted, the discharge current is defined as positive. With this negative correlation, the correlation coefficient can thus vary only between -1 and 0 ; despite this, its absolute value is used for the sake of brevity. Unlike the direction, the strength of a linear relationship is crucial. If two variables have a strong correlation then it is close to 1 . If two variables have a weak correlation then it is close to 0 . In this work, a strong correlation between the voltage and the current indicates a strong linear relationship to estimate the battery resistance. Therefore, the parameter estimates whose correlation coefficient exceeds a certain limit are selected. The limit should be valued around 1; yet, it will be fine-tuned further. By going through this sequence, the determined parameter estimate, that is, the slope in the model can be adapted to changes in the battery resistance.

\section{Verification of Proposed Algorithm}

This section presents the verification of the proposed algorithm for the estimation of the battery resistance. Now that this algorithm is designed with an application in HEVs, a driving cycle that can reproduce their actual operation is used for verification. The dynamic current from the driving cycle is applied to fresh cells such that their internal resistance is estimated. As noted, not all of the parameter estimates are taken into account. Instead, parameter estimates that satisfy the requirements are exclusively chosen. The requirements are meant to identify the battery resistance more reliably. Then, the dynamic current is also given to aged cells and thus their estimated resistance is compared with the fresh cells'.

\subsection{Experimental results}

In this work, the LIB cells are built using the composite 
$\mathrm{LiNi}_{1 / 3} \mathrm{Mn}_{1 / 3} \mathrm{Co}_{1 / 3} \mathrm{O}_{2}-\mathrm{LiMn}_{2} \mathrm{O}_{4}$ (NMC - LMO) cathode material. The cells offer a nominal capacity of $6.5 \mathrm{Ah}$ and maximum three-second charge and discharge pulse powers of $700 \mathrm{~W}$ and $875 \mathrm{~W}$, respectively, at the standard conditions $\left(25^{\circ} \mathrm{C}, 50 \% \mathrm{SOC}\right)$. The cells are thus intended for transient and high power applications primarily including commercial HEVs.

The cell degradation was performed by thermal aging. The cells were stored at the elevated temperature over $50^{\circ} \mathrm{C}$ to accelerate their degradation. Before storing the cells in the hot chamber, baseline performance tests were carried out to measure the capacity, resistance and power of the fresh cells. During thermal aging, the cells were clamped at $50 \%$ SOC and periodically underwent the reference performance tests (RPTs). The RPTs were repeated at every four weeks and this aging campaign continued until 24 weeks. During the RPT, the resistance was measured with the pulse current. This test consists of a three seconds discharge and charge pulse at different current rates ranging from 0.1 to $10 \mathrm{C}$ with a 30 seconds rest period in-between. This test, usually referred to as the hybrid pulse power characterization (HPPC), results in the direct current internal resistance (DCIR). The measured DCIR is used as another battery degradation index with which the resistance estimated from the driving cycle is compared.

The driving cycle is constructed by using recorded data from driving of HEVs, which is specifically the current to and from the battery by charging (when regenerative braking) and discharging (when accelerating). As shown in Fig. 2(a), the driving cycle comes with a 1377 seconds stop-and-go city mode followed by a 763 seconds aggressive highway mode, for a total duration of 2140 seconds. To follow the driving cycle, the current swings from -100 to $150 \mathrm{~A}$ transiently, which causes the cell voltage to go up and down between 3,860 and $3,480 \mathrm{mV}$ and also the SOC to fluctuate around $50 \%$. It is noted that in contrast to the battery for BEVs, the battery used in HEVs tends to cycle about a relatively fixed SOC. The temperature begins at $25^{\circ} \mathrm{C}$ and gradually rises by $7^{\circ} \mathrm{C}$ throughout the driving cycle.

\subsection{Fresh cell test results}

The internal resistance of the fresh cells is estimated based on the dynamic current from the driving cycle. It is noted that the results from three cell samples are averaged out, with little variance throughout the driving cycle. As mentioned, the driving cycle lasting 2,140 seconds produces total 35 sets of data, thereby resulting in a sequence of 35 parameter estimates. To restate, the parameter in the model is identified by using the least squares estimator, namely, the LMS filter. The parameter estimate here is a slope which reflects the cell resistance. During the driving cycle, the cell resistance is estimated to vary from 0.92 to $1.62 \mathrm{~m} \Omega$ (see Fig. 2(a)). Such a large
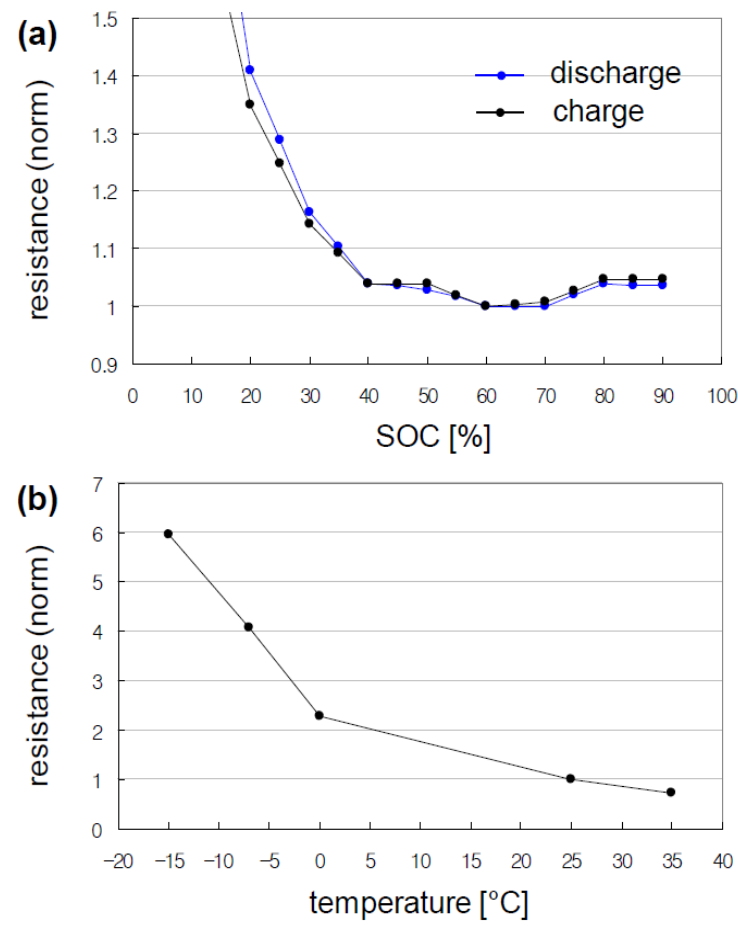

Fig. 3. Dependence of the battery resistance onto (a) SOC (normalized at 60\%) and (b) temperature (normalized at $25^{\circ} \mathrm{C}$ )

deviation in the cell resistance cannot be physically justified; rather, it is unfavorably affected by experimental conditions.

As shown in Fig. 3, it is found that the SOC and temperature are contributing conditions to the battery resistance, thus, to the battery power. To mitigate their adverse effects, the ranges of the SOC and temperature are fixed such that the LMS filter is allowed to run only within the predetermined conditions. The SOC ranging from 60 to $65 \%$ is chosen for minimizing the influence of SOC in that regardless of charge/discharge the DCIR measurement is unchanged within this range. Also, the temperature between 25 and $30^{\circ} \mathrm{C}$ is selected. Due to these limits, 27 sets of data (out of entire 35 data sets) are found to be of no use (see Fig. 4(a) and (b)). Then, the correlation coefficient is calculated from each of 8 remaining sets of data. By trial and error, the limit of the correlation coefficient is set to be 0.86 (see Fig. 4(c)). With this threshold, only two sets of data are revealed to be effective: 10th and 11th. This can be backed by comparing the correlation coefficient. As shown in Fig. 5, compared with the 5th data set, the 10th data set offers a stronger correlation between the voltage and the current, which represents a stronger linear relationship. Therefore, the 10th data set is suitable to estimate the resistance; yet, the 5th data set is not. Consequently, the LMS filter is selectively applied to these qualified data sets, thereby determining the parameter estimates which are the estimated resistance as high as $1.2 \mathrm{~m} \Omega$. The aforementioned sequence is illustrated in Fig. 6. 

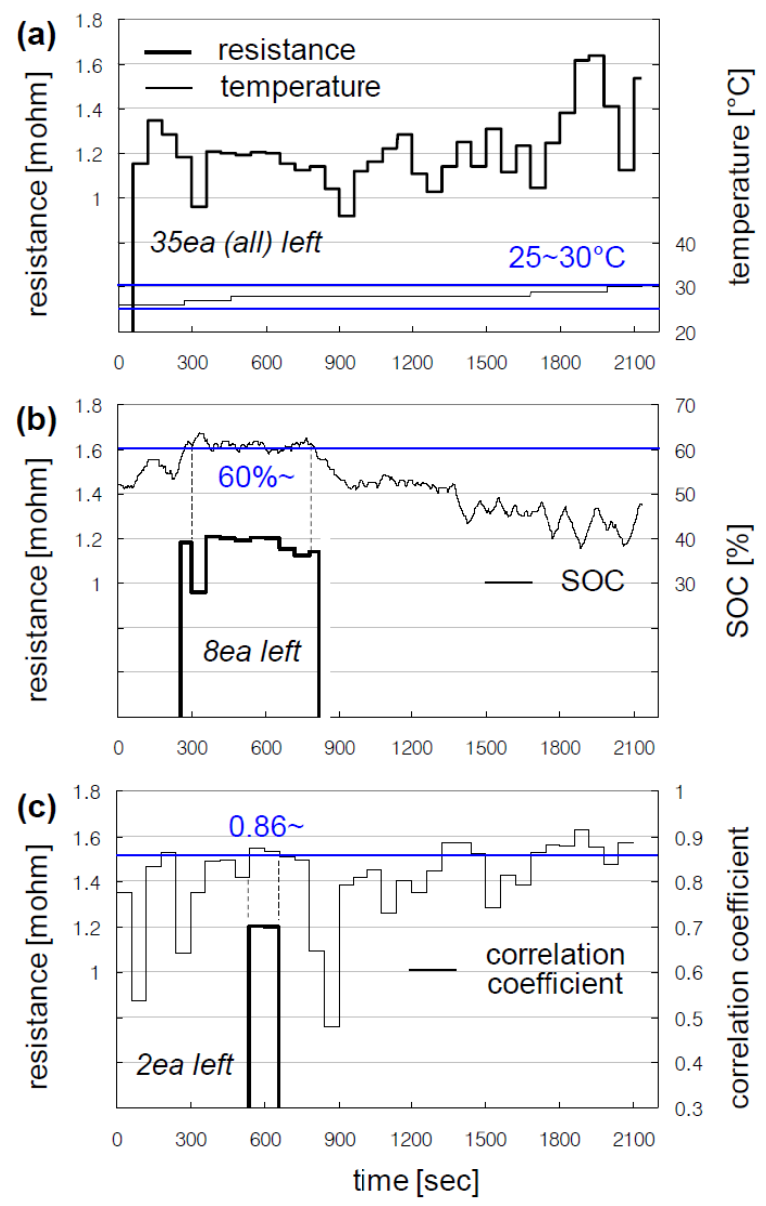

Fig. 4. Sequence performed to select the qualified data sets according to temperature, SOC and correlation
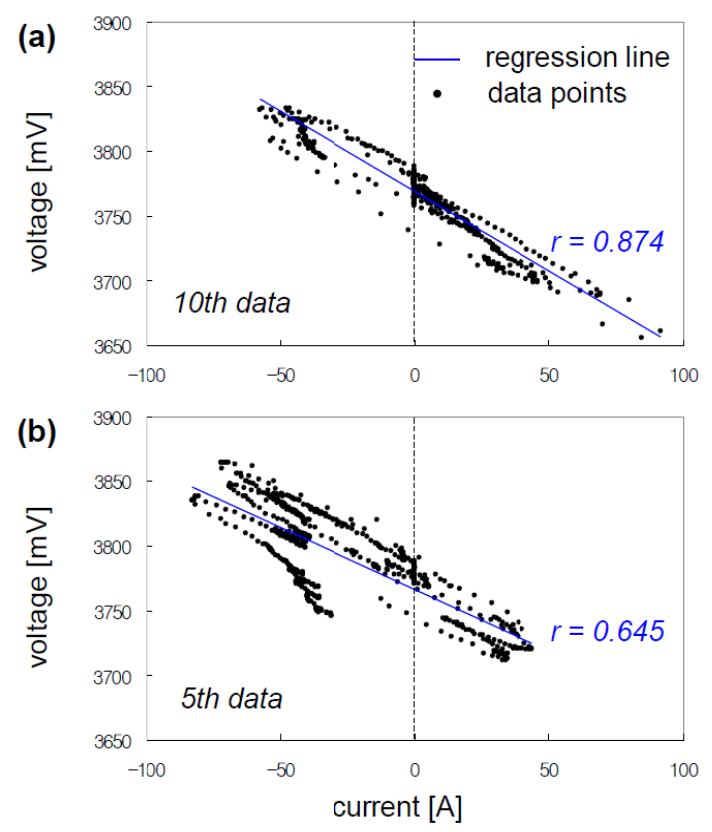

Fig. 5. Comparison of data sets with respect to the correlation coefficient

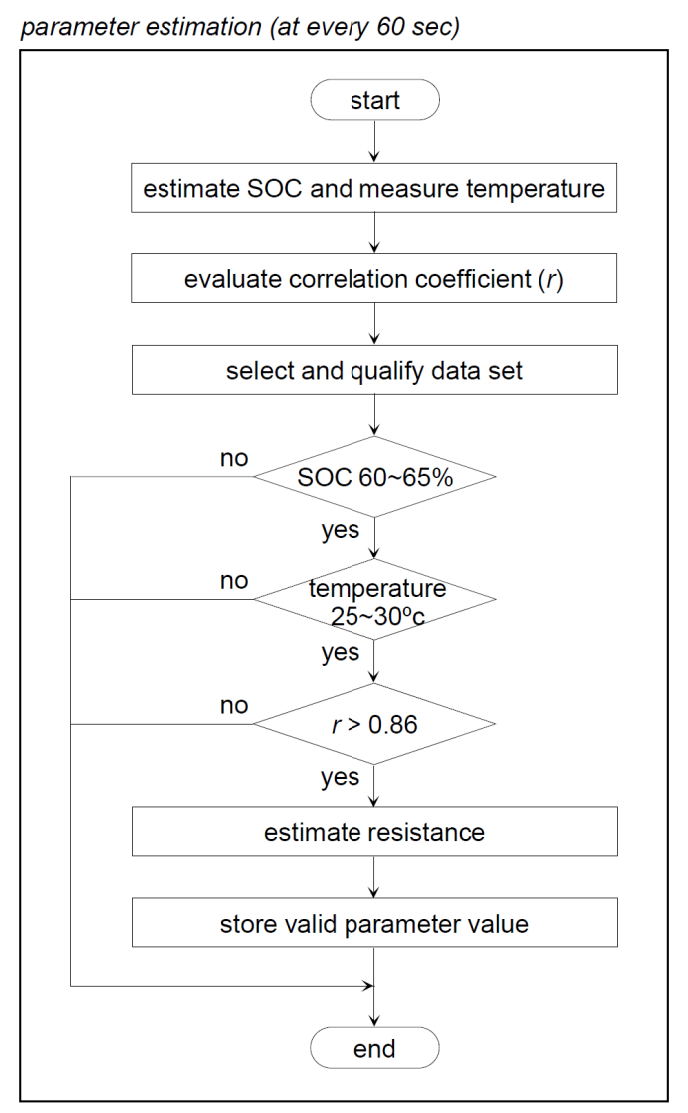

Fig. 6. Operational process of the devised algorithm

\subsection{Aged cell test results}

Next, the same sequence is also applied to the aged cells in order to identify the parameter estimates, expecting their adaptability to changes in the cell resistance. Again, it is noted that the results from three cell samples are averaged out, with negligible variance throughout six RPTs performed during the aging time. It is observed that the capacity decreases monotonically, whereas the resistance increases but with some fluctuations around the 12th week(see Fig. 7(a)). Although the observed dip in the measured DCIR cannot be clearly accounted for, it is also found with another group of cell samples whose degradation is performed by cycling (see Fig. 7(b)). It is noted that cycling is another way of aging with storage. Along with the DCIR measurement, the internal resistance of the aged cells is also estimated based on the dynamic current from the driving cycle. It is turned out that the estimated resistance rises by 2 to $4 \%$ at every month and finally increases by approximately $12 \%$. This result is matched against the DCIR measurement as a function of the aging time (see Fig. 7(c)). Except for the observed dip in the middle, throughout the aging time the measured DCIR is nearly $5 \%$ greater than the cell resistance estimated by using the LMS filter. Despite of their inappreciable difference, this comparison does not mean much. As noted, the DCIR measurement is merely used 


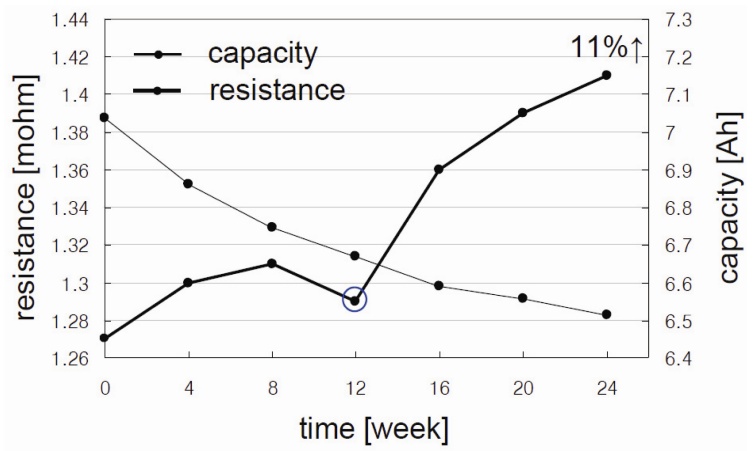

(a)

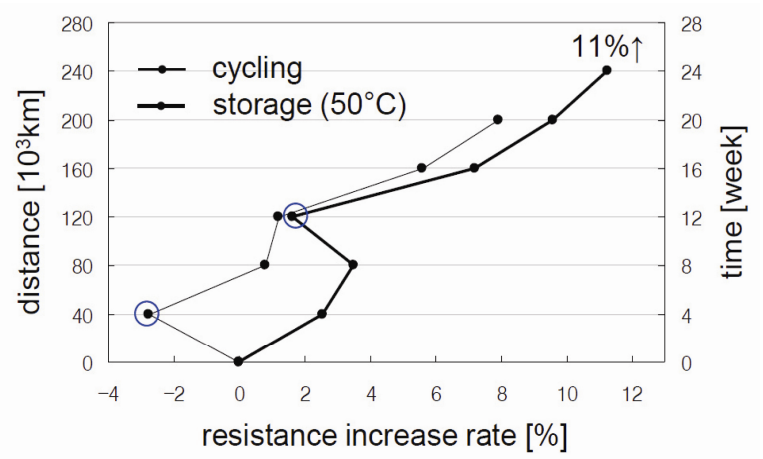

(b)

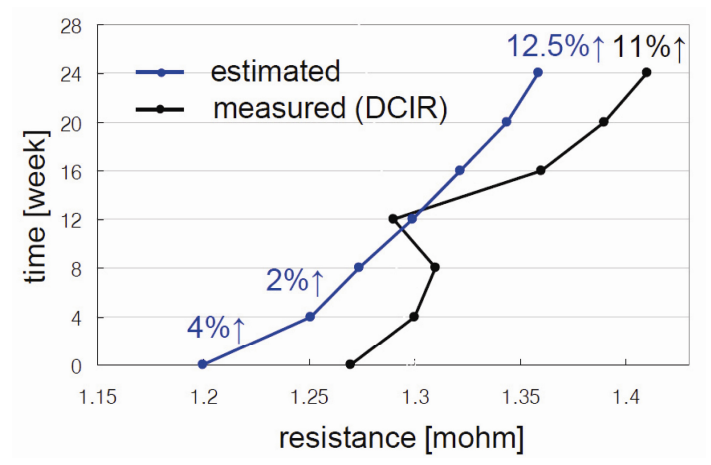

(c)

Fig. 7. RPT results from the aged cells.

here as another battery degradation index that can serve as a reference. Although it has been widely used as a way of measuring the battery resistance, the measured DCIR is very sensitive to experimental conditions given. Rather than this, a comparison among the parameter estimates, specifically, the estimated resistance that is adapted to changes in the cell resistance is crucial.

\section{Conclusion}

This paper proposes a computationally efficient algorithm that can monitor the battery resistance by only using the limited computational resources of the BMS. For this reason, the ordinary least squares estimator is used to identify the parameter in the simple resistance model in the form of a first-order polynomial. These methods used in the devised algorithm are desired for significant advances in computational efficiency which is a key to implementing the parameter estimator into the BMS, along with the battery model. For reliable estimation of the battery resistance, the requirements of the SOC, temperature and correlation coefficient are imposed upon the parameter estimate. Thanks to this, the devised algorithm has the capability to distinguish between the cell resistances which increase by more than $2 \%$. The estimated resistance is validated by matching against the measured resistance (DCIR).

\section{References}

[1] G. L. Plett, "Extended Kalman filtering for battery management systems of LiPB-based HEV battery packs: Part 3. State and parameter estimation," Journal of Power Sources, Vol. 134, pp. 277-292, Aug. 2004.

[2] G. L. Plett, "Sigma-point Kalman filtering for battery management systems of LiPB-based HEV battery packs: Part 2: Simultaneous state and parameter estimation," Journal of Power Sources, Vol. 161, pp. 1369-1384, Oct. 2006.

[3] W. Waag, C. Fleischer, D. U. Sauer, "On-line estimation of lithium-ion battery impedance parameters using a novel varied-parameters approach," Journal of Power Sources, Vol. 237, pp. 260-269, Sep. 2013.

[4] C. Fleischer, W. Waag, H. -M. Heyn, D. U. Sauer, "On-line adaptive battery impedance parameter and state estimation considering physical principles in reduced order equivalent circuit battery models: Part 2. Parameter and state estimation," Journal of Power Sources, Vol. 262, pp. 457-482, Sep. 2014.

[5] M. Verbrugge, B. Koch, "Generalized recursive algorithm for adaptive multiparameter regression: Application to lead acid, nickel metal hydride, and lithium-ion batteries," Journal of Electrochemical Society, Vol. 153 , pp. A187-A201, Jan. 2006.

[6] M. Verbrugge, "Adaptive, multi-parameter battery state estimator with optimized time-weighting factors," Journal of Applied Electrochemistry, Vol. 37, pp. 605-616, Feb. 2007.

[7] S. Wang, M. Verbrugge, J. S. Wang, P. Liu, "Power prediction from a battery state estimator that incorporates diffusion resistance," Journal of Power Sources, Vol. 214, pp. 399-406, Apr. 2012.

[8] A. P. Schmidt, M. Bitzer, A. W. Imre, L. Guzzella, "Experiment-driven electrochemical modeling and systematic parameterization for a lithiumion battery cell," Journal of Power Sources, Vol. 195, pp. 50715080, Aug. 2010.

[9] G. K. Prasad, C. D. Rahn, "Model based identification of aging parameters in lithium ion batteries," Journal of Power Sources, Vol. 232, pp. 79-85, Jun. 2013. 


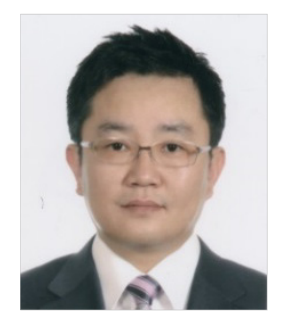

Jin-Ho Park $\mathrm{He}$ received his B.S. degree in Mechanical Engineering from Hanyang University, Seoul, Korea in 1989 and his M.S. in Mechanical Engineering from University of Alabama, Tuscaloosa, AL, USA, in 1991. From 1992 to 1999 , he worked for Mando Machinery Corporation as a research engineer. From 1999 to 2003, he worked for Bosch Electrical Drives Corporation as a senior research engineer. Since 2003, he has worked for Hyundai Motor Company in R \& D Division as a General Manager. His research area is high voltage battery system application into the eco-friendly vehicles including HEV, PHEV, EV and FCEV.

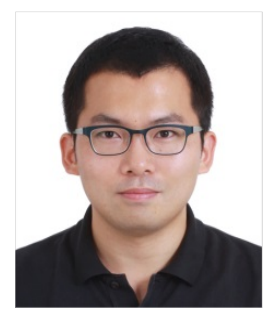

Woo-Suk Sung He received his B.S. degrees in Mechanical and Electrical Engineering from Sungkyunkwan University, Seoul, Korea in 2003 and his M.S. degree in Electrical Engineering from Louisiana State University, Baton Rouge, LA in 2006, respectively. From 2006 to present, he has worked as a research engineer in R\&D Division, Hyundai Motor Company. His research interests include the model-based state estimation and control of lithium-ion batteries for automotive application.

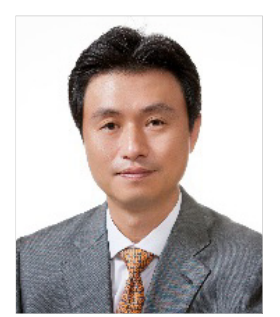

Byoung Kuk Lee He received the B.S. and the M.S. degrees from Hanyang University, Seoul, Korea, in 1994 and 1996, respectively and the Ph.D. degree from Texas A\&M University, College Station, TX, USA, in 2001, all in electrical engineering. From 2003 to 2005, he was a Senior Researcher with Power Electronics Group, Korea Electrotechnology Research Institute, Changwon, Korea. From 2006, he is with the College of Information and Communication Engineering, Sungkyunkwan University, Suwon, Korea. His research interests include on-board charger and wireless power transfer for electric vehicles, energy storage systems, hybrid renewable energy systems, dc distribution systems for home appliances, power conditioning systems for fuel cells and photovoltaic, modeling and simulation, and power electronics. Prof. Lee received the Outstanding Scientists of the 21st Century from IBC and listed on 2008 Ed. of Who's Who in America and 2009 Ed. of Who's Who in the World. He is an Associate Editor in the IEEE Transactions on Industrial Electronics and Guest Associate Editor in the IEEE Transactions on Power Electronics. He was the Presenter for Professional Education Seminar with the topic of "On-Board Charger Technology for EVs and
PHEVs" at the IEEE Applied Power Electronics Conference in 2014 and was the General Chair for the IEEE Vehicular Power and Propulsion Conference in 2012. 